\title{
O NAUTIMODELISMO COMO FERRAMENTA DE EXTENSÃO DO CURSO DE ENGENHARIA NAVAL DA UNIVERSIDADE DO ESTADO DO AMAZONAS
}

Valéria da Silva Costa-vsc.eng@uea.edu.br

Universidade do Estado do Amazonas

Avenida Darcy Vargas, 1200 - Parque 10 de Novembro

CEP: 69035-020 - Manaus - Amazonas

Samuel Pond de Medeiros - spm.eng@uea.edu.br

Universidade do Estado do Amazonas

Avenida Darcy Vargas, 1200 - Parque 10 de Novembro

CEP: 69035-020 - Manaus - Amazonas

Carolina de Oliveira e Castro - cdoc.eng16@uea.edu.br

Universidade do Estado do Amazonas

Avenida Darcy Vargas, 1200 - Parque 10 de Novembro

CEP: 69035-020-Manaus-Amazonas

Resumo: A crescente adesão de alunos de Engenharia Naval da Universidade do Estado Amazonas ao nautimodelismo, que é prática de construção de modelos de embarcação em escala reduzida, trouxe consigo resultados positivos para os discentes inseridos, pois estes tinham acesso à prática e à aprimoração dos conhecimentos adquiridos na universidade. $O$ objetivo deste artigo é analisar a abordagem do nautimodelismo dentro das características de um programa de extensão e avaliar sua eficácia perante o contexto social, cultural $e$ tecnológico discutindo seus benefícios para os participantes. Para esta avaliação foram utilizados como ferramenta metodológica a pesquisa descritiva e a pesquisa documental. A pesquisa descritiva consistiu-se na observação, registro, interpretação e análise das ações praticadas pelos alunos durante o período de participação em equipes de nautimodelismo. Já a pesquisa documental pode validar os efeitos destas ações por meio de dados coletados pelo levantamento de informações a partir de publicações como memoriais de cálculo, artigos, reportagens, relatórios de projeto, entre outros. Os resultados demonstraram a relação entre a prática do nautimodelismo como uma atividade relevante de extensão que auxilia, sob diversos aspectos, ao desenvolvimento dos discentes e por conseguinte, pode-se concluir a importância da promoção e investimento no nautimodelismo como ferramenta para melhorar a qualidade do aprendizado universitário.

Palavras-chave: Nautimodelismo. Projeto de extensão. Engenharia naval. 


\section{INTRODUÇÃ̃O}

$\mathrm{Na}$ Universidade do Estado do Amazonas (UEA), observou-se o crescimento da participação de discentes do curso de engenharia naval em projetos de nautimodelismo, cujo objetivo é a criação de modelos de embarcação em escala reduzida com finalidade de utilizálos em desafios universitários desta categoria. Ao investigar este movimento, pode-se relacionar os projetos de nautimodelismo aos resultados que os discentes apresentavam, seja em criação de inovação, pesquisas e desenvolvimento de ideias, além do desenvolvimento de conhecimentos práticos e extra-curriculares.

Tais resultados se enquadram no que é esperado dentro dos projetos de extensão. Estes, tratam-se de um conjunto de ações contínuas que detém caráter social, cultural, educativo e científico. A extensão é destinada a interação entre a instituição de ensino e a comunidade, seja dentro do âmbito acadêmico ou abertas ao público. (O QUE, 2020)

Logo, tornou-se necessário verificar como os projetos de nautimodelismo funcionam, de fato, para o desenvolvimento do aluno. Este artigo tem como objetivo analisar, por meio da pesquisa de documentos, observação e pesquisa descritiva, a população de alunos de Engenharia Naval participantes destes projetos. A partir desta análise, minuciaram-se quais as implicações decorrentes desta participação nos, expondo o andamento e suas vertentes, a eficiência, resultados e principalmente a relação do nautimodelismo como ferramenta de extensão dentro da própria comunidade acadêmica.

\section{REFERENCIAL TEÓRICO}

\subsection{Projeto de extensão}

O Projeto de Extensão é uma ação contínua de caráter educativo, social e cultural, científico ou tecnológico, com objetivo específico e prazo determinado (PROJETOS DE EXTENSÃO, 2020). O Projeto de Extensão tem com seu principal objetivo a integração dos três pilares da educação superior: a pesquisa, o ensino e a extensão; gerando conexões externas ao campus universitário. Além de possuir um viés social forte visto que um de seus objetivos principais é a interação com a comunidade, o desenvolvimento de projeto que tenha efeito educativo, informativo ou construtivo na comunidade e a enriqueça. (O QUE, 2020)

\subsection{Nautimodelismo}

Segundo Carneiro (2012), a prática do nautimodelismo é tão antiga quanto a história humana, existem réplicas de embarcações datadas há 4000 anos. O nautimodelismo é a prática de construção de modelos de embarcações em pequena escala. $\mathrm{O}$ modelismo naval é dividido em duas categorias: estático e navegável. O estático está relacionado aos modelos de vitrine, mais voltadas para decoração e coleção. O modelismo naval navegável é relacionado a modelos em escalas reduzidas de embarcações já existentes, sendo controladas através de rádios ou não e sendo propulsadas por motores elétricos, a vapor ou apenas através da força dos ventos.

Para a engenharia Naval, o nautimodelismo pode ser pensado a partir da perspectiva de laboratório para as práticas universitárias. Visto que os modelos são uma representação mais facilitada da realidade e o seu uso em testes pode prever o comportamento de diversas

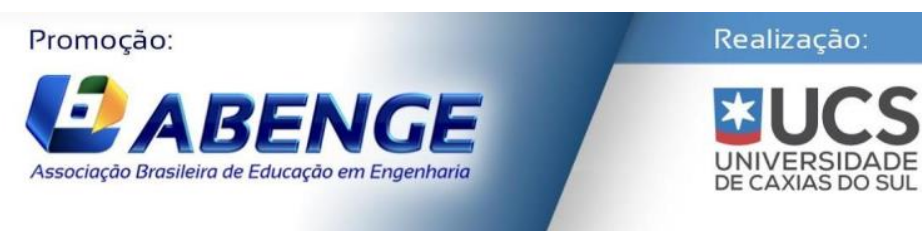


características que o objeto de estudo real pode possuir. O uso destes modelos é vantajoso para a economia de tempo e recursos em projetos, já que auxilia projetistas com o entendimento das características do protótipo e no planejamento de soluções em problemáticas encontradas (CORRÊA, 2017). O teste com modelos se baseia cientificamente no que conhecemos como semelhança ou teoria dos modelos. A teoria dos modelos afirma que é possível prever o comportamento de um protótipo, que é um produto na fase de testes, a partir de seu modelo, desde que haja semelhança geométrica, cinemática e dinâmica entre ambos (CARNEIRO,1993). Dessa forma, o uso destes modelos é vantajoso para a economia de tempo e recursos em projetos, já que auxilia os projetistas com o entendimento das características do protótipo e no planejamento de soluções em problemáticas encontradas. Em outras áreas da engenharia, como a engenharia mecânica e civil, o uso de modelos é amplo pelo mesmo motivo.

Sendo assim, o uso de nautimodelos auxilia em diversos aspectos da formação do engenheiro naval por englobar um grande número de disciplinas que competem ao profissional. Os conceitos aplicados abrangem desde a aplicação da arquitetura naval para os cálculos de estabilidade até a utilização de sistemas de processos construtivos, por vezes se assemelham muito aos processos utilizados em embarcações de grande escala. Também são incluídos os processos mecânicos para a construção do sistema propulsivo e elétricos para que o nautimodelo seja integralmente funcional.

\subsection{Desafio Universitário de Nautidesign (DUNA)}

O Desafio Universitário de Nautidesign é um projeto originado pela Universidade Federal de Joinville em 2013. A proposta do projeto DUNA é a criação de um desafio em que estudantes universitários são estimulados a competir a partir do desenvolvimento e construção de um modelo de escala reduzida de uma embarcação do tipo rebocador, dentro dos requisitos préestabelecidos em edital que será testado em uma série de provas de velocidade, força e manobrabilidade. Outros objetivos são promover a geração de inovações na área, estimular a interação entre os alunos e seus cursos e a prática perante as disciplinas cursadas (QUEM SOMOS, 2020).

\subsection{A engenharia naval na Universidade do Estado do Amazonas}

O curso de engenharia naval na Universidade do Estado do Amazonas (UEA) teve sua primeira turma no ano de 2014, sendo esta um marco para estado que, apesar da tradição no setor naval, jamais teve um curso superior voltado para a área. Até o ano de 2020, cerca de 263 alunos passaram pelo curso (ENGENHARIA NAVAL, 2017). Na condição de curso recém implantado, boa parte de sua estruturação ainda carece de incentivos, principalmente para a construção de laboratórios que permitam o desenvolvimento de novas tecnologias e inovação dentro do ambiente universitário. Pois a universidade tem apenas laboratórios de ambiente virtual disponíveis. Como alternativa as dificuldades encontradas os alunos são motivados a buscar meios que possam servir de complemento ao que é aprendido nas aulas. Daí a grande adesão ao nautimodelismo e ao DUNA visando a oportunidade de aplicação de bases científicas e troca de experiências. 
Por conseguinte, vários pequenos núcleos de alunos dos diversos períodos se uniram em equipes e, atualmente, a universidade conta com quatro equipes participantes do desafio. São elas: Capitão Jack, Jaraqui, Nauticam e Navi. Juntas, chegaram a enviar sete nautimodelos para a competição, sendo então, a universidade com maior número de modelos e alunos participantes do país. Outros projetos fazem parte da área de extensão do curso de engenharia naval, como o projeto LEVIATÃ, cuja proposta é a criação de uma embarcação movida a energia solar que visa o desenvolvimento de tecnologias para utilização de fontes limpas e alternativas de energia.

Tabela 1 - Divisão do número de alunos participantes das equipes de nautimodelismo

\begin{tabular}{|c|c|}
\hline Equipe & Número de alunos \\
\hline Capitão Jack & 25 \\
\hline Jaraqui & 30 \\
\hline Nauticam & 30 \\
\hline Navi & 13 \\
\hline
\end{tabular}

Fonte: Autores, 2020.

\section{METODOLOGIA}

Neste artigo foi utilizada a pesquisa documental e a pesquisa descritiva. Conforme Fonseca (2002), a pesquisa documental baseia-se em fontes de dados diversificadas e primárias, ou seja, aquelas informações que não receberam tratamento científico prévio e são dispersas. Logo, para este fim, foram coletadas informações a partir do levantamento de registros como memoriais de cálculo, relatórios de projeto de extensão enviados à Pró Reitoria de Extensão da UEA, reportagens, documentos oficiais e materiais técnicos produzidos pelas próprias equipes. $\mathrm{O}$ período definido como de interesse para esta coleta foi parametrizado a partir do início da formação da primeira equipe de nautimodelismo, o Jaraqui, até o ano de 2020 em que há, no total, quatro equipes na universidade.

Já a pesquisa descritiva, segundo Gil (2002), sugere caracterizar um fenômeno ao descrever a realidade de forma imparcial. Assim sendo, a linha de ação para a aplicação da pesquisa descritiva neste artigo consistiu em observar, interrogar, coletar, analisar, registrar e interpretar as ações desenvolvidas pelos alunos dentro das equipes de nautimodelismo, do momento da concepção de um projeto básico até a etapa de testes com o modelo construído. Esta sequência de ações foi classificada em três etapas bem diferenciadas, que são:

\section{Desenvolvimento do projeto conceitual e projeto básico}

O projeto conceitual é caracterizado como a etapa do projeto onde é realizada a elaboração e definição dos interesses e objetivos básicos do nautimodelo, baseando-se em especificações pré-estabelecidas pela equipe dentro do escopo do edital apresentado pelo DUNA, como 
comprimento de boca (largura do modelo), comprimento, pontal (altura ao centro do casco), peso mínimo, forma do casco, entre outros.

O projeto básico é o passo seguinte ao conceitual, nele as diretrizes do projeto conceitual são refinadas e somam-se novas alterações ao projeto. Durante todas as etapas temos a inserção da participação de alunos de outras áreas da engenharia, que tem por objetivo promover o desenvolvimento de novas tecnologias e estudos preliminares, em conjunto, que tornem o modelo mais próximo do esperado. Como exemplo, podemos citar a participação de alunos de engenharia mecânica que trabalham em parceria aos alunos de engenharia naval para que o projeto do sistema propulsivo tenha o melhor aproveitamento e o mínimo de perdas.

\section{Captação de recursos e Gerenciamento}

Realizada paralelamente ao item anterior, a captação de recursos visa a reunião de recursos que tornem a construção do nautimodelo viável e visibilidade para a equipe. Nesta etapa os alunos são motivados a buscar parceiros dentro e fora do ambiente universitário. Para isso, realizam pesquisas a cerca de fornecedores de materiais e serviços que possam ser úteis para o projeto e visitas a empresas para firmar patrocínios e/ou apoios para a equipe. Além da participação em feiras e congressos, apresentando os nautimodelos para exposição e por conseguinte realizando a divulgação do conhecimento técnico e prático.

Também nesta etapa há o gerenciamento do projeto, incluindo os custos, cronogramas de trabalho, riscos de investimento e mais. Já que o valor arrecadado pela captação deve ser utilizado de forma hábil para que seja o suficiente para atender as necessidades do projeto dentro do tempo estimado, garantindo que todos os materiais e serviços estejam disponíveis de acordo com o que é apontado pelo cronograma.

\section{Manufatura e testes}

Mediante a revisão do projeto básico que é novamente refinado, se dá origem ao projeto executivo do modelo. O projeto executivo, de acordo com o que é apresentado pelo Manual de Obras Públicas (2014), é o conjunto de informações técnicas que são necessárias para a realização de um empreendimento e deve conter de forma completa e precisa todas as indicações necessárias e detalhes construtivos para a construção, instalação e montagem de um objeto.

A partir das instruções do projeto executivo, o modelo entra na fase de manufatura, ou seja, o momento em que transforma-se a matéria-prima em um bem acabado e para isto associam-se os materiais, métodos construtivos definidos, o gerenciamento de custos e principalmente o cronograma. Esta é a etapa do desenvolvimento que demanda maior atenção dos alunos por seu alto grau de complexidade e precisão, além de demandar dos participantes um grau avançado de conhecimentos práticos. Com a manufatura concluída e os equipamentos devidamente montados, inicia-se a fase de testes. Nela a equipe põe a prova o projeto, analisando os resultados e comparando-os com os resultados esperados no projeto básico.

Pela análise do material recolhido pela pesquisa descritiva, foi possível obter dados suficientes para entender quais as ações realizadas pelos estudantes durante a participação em um projeto e validar as consequências destas ações por meio da pesquisa documental. Assim, pode-se confrontar a hipótese de que o nautimodelismo é benéfico ao estudante e funciona como 
ferramenta de extensão do curso de engenharia naval, e como resultado obtivemos que a hipótese é válida e correta. Ou seja, o nautimodelismo proporciona o que as bases de um projeto de extensão exigem ao fornecer uma série de benefícios profissionais, acadêmicos e pessoais aos seus participantes.

\section{RESULTADOS}

\subsection{Parceria entre a universidade e empresas}

Na UEA, após o levantamento de dados, constatou-se que as equipes de nautimodelismo captaram 38 empresas para parcerias, as quais foram responsáveis pelos mais diversos tipos de colaborações. Na Tabela 2, o número de empresas é apresentado de acordo com a equipe parceira.

Tabela 2 - Divisão do número total de empresas

parceiras à equipes no período 2015-2020.

\begin{tabular}{|c|c|}
\hline Equipe & Empresas Parceiras \\
\hline Capitão Jack & 5 \\
\hline Jaraqui & 13 \\
\hline Nauticam & 10 \\
\hline Navi & 10 \\
\hline
\end{tabular}

Fonte: Autores, 2020.

A parceria entre os alunos participantes e as empresas nasce da necessidade de captar recursos para o desenvolvimento do projeto. Este tipo de parceria é importante e benéfico para ambos os lados, já que são geradores de oportunidades.

Para os alunos é a oportunidade de estabelecer vínculos com um patrocinador ao divulgar um produto ou marca durante a competição em troca de auxílio. Em geral, as empresas procuradas para esta troca são aquelas já inseridas dentro do setor da engenharia ou que sejam interessantes para o projeto. Sendo assim, acabam por aproximar o aluno de fornecedores de matéria-prima e serviços, o que é um reforço positivo para que o aluno saia da universidade já inserido no mercado. A parceria abre as portas das empresas para os alunos, recebendo-os para a troca de experiências, estimulando conhecimentos mais especializados. Para as empresas, a colaboração com a universidade é benéfica para o estreitamento de laços e serve, muitas vezes, como ferramenta de recrutamento para empresas, que podem selecionar os alunos que se tornam mais relevantes e trazê-los para seu quadro de empregados como aposta de bons profissionais. Há, de igual forma, o intercâmbio de ideias que contribuem para a renovação e atualização dos funcionários. Ademais, esse também é o acesso das empresas à novos resultados de pesquisas que são bases para a origem de novas ideias de produtos e/ou serviços para o mercado.

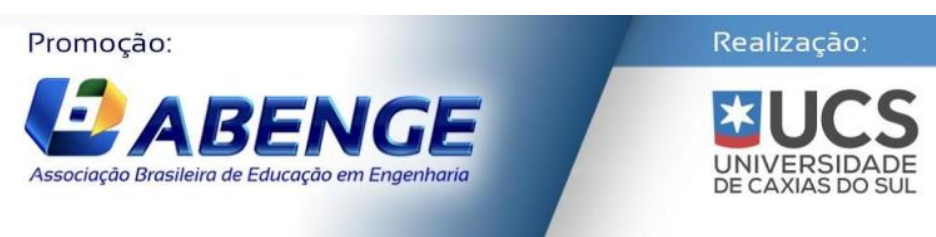




\subsection{Geração de Inovação e Produções científicas}

A busca por embarcações mais eficientes, leves e sustentáveis para que as equipes possuam melhor rendimento nas competições acadêmicas, é a porta de entrada para a pesquisas sobre modelagem de casco, projeto de hélices, estabilidade, entre outros.

Dentre as inovações apresentadas pelos acadêmicos, destaca-se, a pesquisa na utilização de novos materiais, inclusive de origem natural, para a manufatura de embarcações, como justifica GONÇALVES et al. (2018) que questões referentes ao uso e esgotamento dos recursos naturais, está fazendo com que a indústria priorize a utilização de materiais naturais, podendo ser aplicada na construção civil, naval, indústria têxtil, na área da saúde, etc. Para estudar as características e o comportamento de fibras vegetais na construção de embarcações, a equipe NAVI aplicou em 2018 a fibra de curauá e em 2019 a fibra de juta na manufatura das embarcações construídas para o Desafio Universitário de Nautidesign. Os estudos, métodos construtivos e resultados da utilização dessas fibras naturais, foram apresentados à PROGEX/UEA.

As pesquisas voltadas à melhoria das embarcações participantes das competições, renderam também aos acadêmicos publicações em importantes congressos de engenharia. Dentre os temas eles destacam-se estabilidade e construção de embarcações fotovoltaicas. No primeiro caso, tem-se o Estudo da Influência de Bolinas em Embarcações Fluviais (MAGALHÃES et al., 2015) apresentado no $9^{\circ}$ Seminário de Transporte e Desenvolvimento Hidroviário Interior, utilizou o rebocador em pequena escala para comparar e medir as oscilações da embarcação sem e com o sistema de amortecimento utilizando chapas de alumínio naval. A construção do catamarã movido a energia solar, concedeu aos alunos de engenharia naval a apresentação do artigo Construção de uma Embarcação Solar para Competição Universitária (ARAÚJO et al., 2016) no XLIV Congresso Brasileiro de Educação em Engenharia, no qual descreve os procedimentos, materiais, métodos e dificuldades enfrentadas pela equipe para a construção do barco com energia fotovoltaica para o Desafio Solar Brasil. No $27^{\circ}$ Congresso Internacional de Transporte Aquaviário, Construção Naval e Offshore foi apresentado Aplicação de proposta de abordagem prática para seleção preliminar de propulsores da série B-Troost para um catamarã solar (OLIVEIRA et al., 2018), em que o consistiu no desenvolvimento de uma abordagem alternativa para propulsores navais, utilizando o software MATLAB®, eliminando os erros de cálculo e reduzindo o tempo de dimensionamento do propulsor em relação ao método tradicional.

\subsection{Interdisciplinaridade}

Com a necessidade de ultrapassar os limites acadêmicos, os projetos de nautimodelismo suscitaram a interdisciplinaridade. Já que torna-se necessário, assim como em qualquer âmbito profissional, que o sujeito não seja engessado somente em sua área, mas que troque conhecimentos e vivências para adquirir múltiplos conhecimentos, podendo acrescentá-los em seu currículo como diferencial perante aqueles que não se propuseram à mesma estratégia. $\mathrm{O}$ mesmo ocorre com os alunos, que por vezes, acabam se associando a alunos de outros cursos -

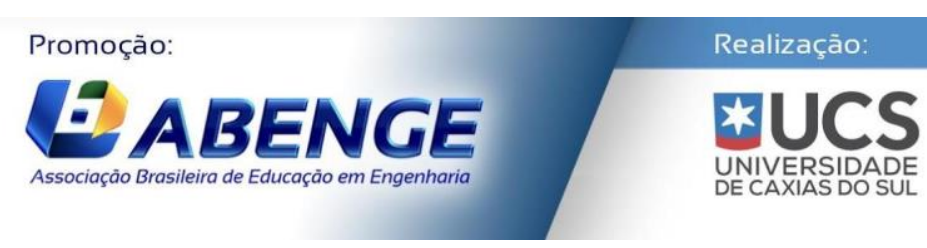


até mesmo de fora da engenharia - em busca de novas ideias e de soluções para seus problemas práticos.

\section{DISCUSSÃO}

Os resultados oriundos da pesquisa descritiva associados aos dados da pesquisa documental demonstraram o desenvolvimento dos alunos ao serem avaliados sob a perspectiva das bases que os programas de extensão apresentam. As principais vertentes avaliadas foram: o eixo social, eixo científico e o eixo cultural. Estes aspectos são aqueles incluídos no que é apresentado como as principais características de um projeto de extensão.

No eixo social temos o sistema de parceria entre empresa e universidade, que promove a transposição de barreiras que dificultam o acesso dos alunos ao mercado, além, há a troca de conhecimentos entre os alunos e a população de trabalhadores externos ao ambiente universitário. No eixo cultural, observamos a implantação, cada vez mais estruturada - ainda que de forma pouco estudada -, da interdisciplinaridade, que é fundamental para que os alunos saiam da universidade cada vez mais aptos a trabalhar em um mundo globalizado e multifacetado. Por fim, no eixo científico, há ganhos enormes para a pesquisa realizada pela UEA, ainda mais, quando se percebe que os alunos se tornam pesquisadores naturalmente, motivados unicamente pelo desejo de desenvolver um modelo que garanta bons resultados. Por fim, os resultados demonstram que o nautimodelismo pode ser considerado uma metodologia para aplicação dos conceitos de extensão dentro da universidade e comunidade.

Verificou-se também que é possível realizar mais pesquisas sobre os ganhos científicos relacionados ao nautimodelismo e aos projetos de extensão em si, catalogando todos os materiais técnicos-científicos produzidos.

\section{CONCLUSÃO}

A difusão e o aprimoramento do conhecimento são os principais objetivos que qualquer aluno inserido em programas de extensão pode alcançar. Com o advento da inserção do nautimodelismo dentro do curso de engenharia naval foi possível identificar os mesmos objetivos sendo atingidos, ainda que de forma tímida e não oficial. Após este estudo, podemos comprovar a aplicabilidade do nautimodelismo como projeto trouxe os mesmos benefícios esperados a qualquer projeto de extensão implantado. Logo, há relação direta entre ambos e isto demonstra a importância da promoção e do investimento em projetos como ferramenta para melhorar a qualidade do aprendizado universitário. 


\section{REFERENCIAS}

\section{Livros:}

CARNEIRO, F. Análise Dimensional e Teoria da Semelhança dos Modelos Físicos. $1^{\text {a }}$ Ed. Rio de Janeiro: UFRJ, 1993.)

FONSECA, J. J. S. Metodologia da pesquisa científica. Apostila. Fortaleza: UEC, 2002.

GIL, A. C. Como elaborar projetos de pesquisa. $4^{\text {a }}$ Ed - São Paulo: Atlas, 2002.

TRIBUNAL DE CONTAS DA UNIÃO. Secretaria-geral de Controle Externo. Secretaria de Fiscalização de Obras de Infraestrutura Urbana. Manual de Obras Públicas: Recomendações básicas para a contratação e fiscalização de obras de edificações públicas. $4^{\mathrm{a}}$ Ed. Brasília, 2014

\section{Artigos de periódicos:}

GONÇALVES, F. A., AMARAL, E. L. S., \& LOPES, J. L. Fibras Vegetais: Aspectos Gerais, Aproveitamento, Inovação Tecnológica e uso em Compósitos.

\section{Trabalhos em eventos}

MAGALHÃES, Lucas H. S., GRIMM, A. B., Aguiar Filho, B. M., Souza Júnior, J. A., MESQUITA, V. G. S., SOARES, F. J. A. Estudo da Influência de Bolinas em Embarcações Fluviais. $9^{\circ}$ Seminário de Transporte e Desenvolvimento Hidroviário Interior Manaus, 6 a 8 de Outubro de 2015

ARAUJO, Carla P. M., AGUIAR, D. S., SOUZA, R. O. S., LIMA, A. F., CHIRANO, C. V. A. R., MELO, Charles L. S. Construção de uma embarcação solar para competição universitária. COBENGE 2016 XLIV CONGRESSO BRASILEIRO DE EDUCAÇÃ̃O EM ENGENHARIA

OLIVEIRA, Renato A. S. O., CARVAlHO, Paulo G. S., ONETY, R. E., LOPES, A. P. Aplicação de proposta de abordagem prática para seleção preliminar de propulsores da série B-Troost para um catamarã solar. $27^{\circ}$ Congresso Internacional de Transporte Aquaviário, Construção Naval e Offshore

\section{Internet:}

CARNEIRO, Gustavo. Nautimodelismo foi categoria pioneira:Prática começou com os egípcios há 4 mil anos. Rudge Ramos Jornal, São Paulo, 20 de abr. de 2012. Disponível em: <http://www.metodista.br/rronline/rrjornal/nautimodelismo-foi-categoria-pioneira $>$. Acesso em: 7 de mai. de 2020

ENGENHARIA NAVAL. Universidade do Estado do Amazonas, Manaus, 14 de mai. de 2017. Disponível em: <http://cursos1.uea.edu.br/index.php?dest=info\&curso=110>. Acesso em: 22 de mai. de 2020. 
O QUE é um projeto de extensão e qual é sua importância?. UNIDERP, São Paulo, 15 de mai. de 2020. Disponível em: <https://blog.uniderp.com.br/projeto-de-extensao/>. Acesso em: 17 de jun. de 2020.

PROJETOS DE EXTENSÃO. Universidade do Estado do Amazonas, Manaus. Disponível em: < https://proex.uea.edu.br/projetos-de-extensao/>. Acesso em: 22 de mai. de 2020.

QUEM SOMOS. DUNA - Desafio Univesitário de Nautidesign, Santa Catarina, 01 de jan. de 2020. Disponível em: 〈https://www.oficialduna.com/sobre>. Acesso em: 15 de jun. de 2020.

Notas de aula:

CORRÊA, A. Arquitetura naval II. Notas de Aula Universidade do Estado do Amazonas, Manaus: 05 ago. 2017- 12 dez. 2017.

\title{
NAUTIMODELISM AS A EXTENSION TOOL IN THE NAVAL ENGINEERING COURSE OF THE UNIVERSITY OF THE STATE OF AMAZONAS
}

\begin{abstract}
The rising enrollment of Naval Engineering students of the University of State of Amazonas in Nautimodelism, that is the construction of ship models of reduced size, has brought positive results for the enrolled students, because they have access to practice and betterment of the knowledge acquired in the university. This article objective is to analyze the practice of nautimodelism as an extension program and evaluate its efficacy within the social, cultural and technological context and discuss the benefits that it brings to the participants. This evaluation will be made as a methodological tool to the descriptive and documental research. The descriptive methodology consists in the observation, register, interpretation and analysis of the actions taken by the students during the participation period in the nautimodelism teams. The documental research can validate the effects of those actions from data collected by information taken from publications such as calculus memorials, articles and extension reports, among others. The results showed correlation between the nautmodelism practice and the development of the student, and when can conclude the importance of the promotion and investment of such practices as a tool to improve the quality of the university experience.
\end{abstract}

Keywords: Extension project, Nautimodelism, Naval Engineering. 